\section{COPD: Ansprechen auf inhaltive Kortikosteroide vorhersagen}

Bafadhel $\mathrm{M}$ et al. Predictors of exacerbation risk and response to budesonide in patients with chronic obstructive pulmonary disease: a posthoc analysis of three randomised trials. Lancet Respir Med 2018; 6: 117-126

Eine große Posthoc-Analyse von drei randomisiert-kontrollierten Doppelblindstudien zu einer BudesonidFormoterol-Fixkombination im Vergleich zur Formoterol-Gabe alleine untersuchte die Rolle der Eosinophilie als Prädiktor bei chronisch obstruktiver Atemwegserkrankung (COPD). Untersucht wurden die Assoziationen von Eosinophilen im peripheren Blut und dem Exazerbationsrisiko ohne und mit einer Therapie mit inhalativen Kortikosteroiden (ICS).

Die Studien hatten die ICS-FormoterolKombination bei Patienten mit COPD und Exazerbationen in die Anamnese untersucht. Eosinophilenzahlen waren kein Ein- oder Ausschlusskriterium gewesen, deshalb konnte der Analyse ein breites Spektrum von Eosinophilenzahlen im peripheren Blut zugrunde gelegt werden, wie Mona Bahafdel von der Abteilung für Atemwegserkrankung der Universitätsklinik in Oxford und Kollegen berichten. Patienten mit Asthma in der Vorgeschichte waren von der Analyse ausgeschlossen.

\section{Ergebnisse}

Insgesamt konnten Daten von 4528 Patienten ausgewertet werden. Die negative binominale Regressionsanalyse mit Adjustierung für die Expositionszeit und das jeweilige Studiendesign ergaben einen nicht linearen Anstieg der jährlichen Exazerbationsrate mit ansteigender Eosinophilenzahl, wenn Patienten Formoterol alleine erhielten. Bei $\geq 0,10 \times$ 109 Eosinophilen/I zeigte sich ein signifikanter Therapieeffekt im Sinne einer Exazerbationsreduktion durch BudesonidFormoterol gegenüber Formoterol alleine. Die Verhältnisrate (Rate Ratio) be- trug 0,75 (95\% Konfidenzintervall [KI] 0,57-0,99; PInteraktion $=0,015$ ).

Es zeigten sich ebenfalls Interaktionen zwischen Eosinophilenzahl und weiteren Messgrößen der Therapieeffektivität von Kombinations- gegenüber Monotherapie: bei dem Gesundheitsstatus nach dem St-George's-Respiratory Questionnaire $\left(P_{\text {Interaktion }}=0,0043\right.$ ) sowie bei der Einsekundenkapazität (FEV1) vor Bronchodilator-Gabe (linearer Effekt $\mathrm{p}<$ 0,0001, PInteraktion $=0,067$ ). Nur Eosinophilenzahl und Rauchstatus waren unabhängige Prädiktoren des Ansprechens auf die ICS-haltige Kombination im Sinne einer Exazerbationsreduktion (Eosinophilenzahl $P_{\text {Interaktion }}=0,013$; Rauchstatus $\left.P_{\text {Interaktion }}=0,015\right)$. Den ausgeprägtesten Behandlungseffekt zeigten entsprechend aktuelle Raucher mit hohen Eosinophilenzahlen im peripheren Blut.

\section{FAZIT}

Die Autoren plädieren dafür, die Eosinophilenzahlen in den SCOPEXRisikoscore mit aufzunehmen, um Patienten mit hoher Wahrscheinlichkeit zukünftiger Exazerbationen identifizieren zu können. Zusätzlich sollte auch die Zahl der bereits durchgemachten Exazerbationen in den Index eingehen, um ein hohes Risiko für häufige Exazerbationen einzubeziehen. Mit dem erweiterten SCOPEX lassen sich so Patienten identifizieren, die von einer ICS-Therapie im Sinne einer Exazerbationsreduktion profitieren.

Friederike Klein, München 